\title{
Remote ischemic preconditioning does not induce activation of Akt and STAT5 in the rat heart
}

\author{
ANNIKA RAUPACH $^{1}$, KATHARINA FEIGE $^{1}$, CHRISTIAN REITER $^{1}$, TIMO BRANDENBURGER $^{1}$, NICOLE HEINEN $^{1}$, \\ ANDRÉ HEINEN $^{2}$, MARKUS W. HOLLMANN ${ }^{3}$, RAGNAR HUHN ${ }^{1}$ and CAROLIN TORREGROZA ${ }^{1}$ \\ ${ }^{1}$ Department of Anesthesiology, University Hospital Duesseldorf, D-40225 Duesseldorf; \\ ${ }^{2}$ Institute of Cardiovascular Physiology, Heinrich-Heine-University Duesseldorf, D-40225 Duesseldorf, Germany; \\ ${ }^{3}$ Department of Anesthesiology, Amsterdam University Medical Center (AUMC), \\ Location AMC, 1100 DD Amsterdam, The Netherlands
}

Received July 6, 2020; Accepted September 22, 2020

DOI: $10.3892 /$ etm.2021.9849

\begin{abstract}
Remote ischemic preconditioning (RIPC) is hypothesized to be a promising cardioprotective strategy to protect hearts against ischemia and reperfusion (I/R) injury; however, the current understanding of the underlying signal transduction pathways involved remains unclear. It has been previously demonstrated that protein kinase B/AKT, which is a crucial protein of the reperfusion injury salvage kinases pathway, and STAT5, which is a member of the survivor activating factor enhancement pathway, serve a pivotal role in cardioprotection. However, whether and at what time-points (TPs) RIPC leads to the activation of AKT and STAT5 in a rat model of RIPC and I/R injury remains to be determined. The present study hypothesized that RIPC may induce the phosphorylation of AKT and/or STAT5 immediately following RIPC and/or at a later TP with or without subsequent I/R. In the first set of experiments (part A), male Wistar rats were randomized into 2 groups ( $n=6$ per group): The first group underwent RIPC via a hind limb tourniquet $(4 \times 5 \mathrm{~min}$ $\mathrm{I} / \mathrm{R}$ episodes), while the second group received the respective sham treatment. In the second set of experiments (part B), the rats were randomized into 4 groups ( $\mathrm{n}=6$ per group) that either underwent RIPC or sham treatment prior to $35 \mathrm{~min}$ of ischemia by occlusion of the left anterior descending coronary artery followed by $120 \mathrm{~min}$ reperfusion or a respective sham treatment. At the end of the experiments, the heart tissue was isolated in order to analyze the phosphorylation levels of AKT and STAT5.
\end{abstract}

Correspondence to: $\mathrm{Dr}$ Katharina Feige, Department of Anesthesiology, University Hospital Duesseldorf, Moorenstr. 5, D-40225 Duesseldorf, Germany

E-mail: KatharinaKristina.Feige@med.uni-duesseldorf.de

Abbreviations: AAR, area of risk; I/R, ischemia and reperfusion; JAK, Janus kinase; LAD, left anterior descending coronary artery; RIPC, Remote ischemic preconditioning; RISK, reperfusion injury salvage kinases

Key words: remote ischemic preconditioning, Akt, STAT5, ischemia/reperfusion, cardioprotection
The results revealed that RIPC did not induce the immediate or late phosphorylation of AKT or STAT5. In addition, following $\mathrm{I} / \mathrm{R}$, the activation of AKT and STAT5 was not modulated by RIPC. In conclusion, the findings of the present study suggested that RIPC-induced cardioprotection may not be mediated by the activation of AKT or STAT5 at the investigated TPs.

\section{Introduction}

Ischemic heart disease remains the most common cause of death worldwide, accounting for up to $23 \%$ of global deaths (1). To minimize the myocardial damage following acute ischemia, early reperfusion is of crucial importance (2). Unfortunately, reperfusion itself is deleterious for the heart and causes ischemia reperfusion (I/R) injury (2). Cardioprotective interventions, such as ischemic and pharmacological preconditioning, are powerful measures to confer cardioprotection against myocardial I/R injury (3). Ischemic preconditioning (IPC) is performed through the exposure to short cycles of ischemia followed by reperfusion directly at the target organ (4). However, to date, the translation of IPC into the clinic has failed due to the invasiveness of the procedure. Nonetheless, this limitation was discovered to be circumvented through using non-invasive remote IPC (RIPC), which works by applying short cycles of $\mathrm{I} / \mathrm{R}$, for example at a limb, via a blood pressure cuff (5). Although promising experimental results have been published following years of research on RIPC, the exact underlying mechanism of RIPC-induced cardioprotection is still not fully understood (6). The proposed mechanism includes the generation of humoral factors in the remote organ, which can then enter the blood stream to activate and induce protective signaling cascades in the heart (5).

It was previously demonstrated that conditioning strategies protected the myocardium by activating the reperfusion injury salvage kinases (RISK) pathway (7); this pathway mediates cell survival through various signaling cascades, including the activation of endothelial nitric oxide synthase, protein kinase G, PI3K and AKT. Li et al (8) reported that members of the RISK pathway were involved in RIPC-induced cardioprotection, while the PI3K inhibitor wortmannin abolished the cardioprotective effect in a combined in vivo/in vitro model. In addition, increased AKT phosphorylation levels were observed 
in tissues 15 min after RIPC treatment compared with the control group. However, to the best of our knowledge, whether RIPC-induced AKT phosphorylation is merely transient or maintained during $\mathrm{I} / \mathrm{R}$ remains unclear.

Furthermore, previous research has demonstrated that conditioning with various stimuli protects the heart from I/R injury via activation of the survivor activating factor enhancement pathway, which involves TNF $\alpha$, TNF receptor type 2 (TNFR2), Janus kinase (JAK) and STAT3 (9). The tyrosine phosphorylation of STATs by JAKs leads to the dimerization and subsequent translocation of the respective STATs into the nucleus or mitochondria to regulate the expression levels of its target genes, which are primarily involved in energy metabolism (10). Heusch et al (11) identified that the phosphorylation levels of RISK pathway members or STAT3 were increased in both RIPC-treated and control patients before undergoing myocardial intervention, whereas the phosphorylation levels of STAT5 were only upregulated in the RIPC group. While the results from one previous study suggested the involvement STAT5 in RIPC-induced cardioprotection (12), other previous studies have indicated that the phosphorylation of STAT5 may not be involved $(13,14)$. Therefore, the role of STAT5 in RIPC-induced cardioprotection remains unexplained.

As AKT and STAT5 are considered to serve an important role in the signaling pathways of different cardioprotective strategies, the present study aimed to investigate the phosphorylation levels of these key mediators in RIPC-induced cardioprotection in rat hearts in vivo. The current study hypothesized that RIPC may induce the phosphorylation of AKT and/or STAT5 at both time-points (TPs), that is, immediately after RIPC and/or after I/R.

\section{Materials and methods}

Ethics approval. The current study was conducted in accordance with the Guide for the Care and Use of Laboratory Animals published by the National Institutes of Health (publication number 85-23, revised 1996) and the experiments were performed after obtaining ethical approval from the State Agency for Nature, Environment and Consumer Protection, North-Rhine Westphalia, Germany (approval no. 8.87-50.10.37.09.148), and this study comply with the ARRIVE guidelines (15). The animals used were obtained from the breeding facility at the Central Animal Research Facility of the Heinrich-Heine-University Duesseldorf.

Surgical preparation. In part A of the present study, heart tissues were used from rats exclusively treated during the present study. In part B, the heart tissues were obtained from animals used in a previous study by Brandenburger et al (16), which demonstrated a significant reduction in infarct size following RIPC compared with the control hearts.

Surgical preparation was performed as described previously (16). Briefly, male Wistar rats were anesthetized by intraperitoneal sodium pentobarbital injection $(60 \mathrm{mg} / \mathrm{kg}$ body weight). Following tracheal intubation, mechanical ventilation was performed with $30 \%$ oxygen $/ 70 \%$ nitrogen and monitored by blood gas analysis throughout the experiments to maintain the acid-base state within the physiological limits. Anesthesia was maintained by continuous infusion of pentobarbital $(40 \mathrm{mg} / \mathrm{kg} / \mathrm{h})$ and the body temperature was maintained at $37-37.5^{\circ} \mathrm{C}$ using a heating pad. Lateral left-sided thoracotomy was performed by looping a ligature (5-0 Prolene; Ethicon, Inc.; Johnson \& Johnson) around the left anterior descending (LAD) coronary artery to tighten and occlude the coronary artery to induce ischemia. Following successful implementation of the surgical procedures, all animals were allowed to stabilize for $10 \mathrm{~min}$ prior to the start of the respective experimental protocol.

\section{Experimental protocol}

Part A. Part A was performed to investigate the activation of AKT and STAT5 immediately following RIPC. Therefore, the hearts required for the analysis were harvested 10 min after sham or RIPC treatment (TP 1:60 min). The rats (weight, $280 \pm 27 \mathrm{~g}$ ) used for these experiments were randomly assigned into one of two experimental groups (n=6/group; Fig. 1): i) Sham early TP (TP1) group, in which the rats received sham I treatment; and ii) RIPC TP1 group, in which rats received RIPC treatment with 4 cycles of bilateral hind limb ischemia for 5 min interspersed with $5 \mathrm{~min}$ of reperfusion. RIPC treatment was performed by bilateral hind limb ischemia, which was induced by inflating modified blood pressure cuffs to $200 \mathrm{mmHg}$, followed by the initiation of reperfusion by deflating the cuffs. For the sham I treatment, the cuffs were placed on the hind limbs, but not inflated. Following $10 \mathrm{~min}$ of the respective treatments, the heart was excised, frozen in liquid nitrogen and stored at $-80^{\circ} \mathrm{C}$ for further analysis.

Part B. Part B experiments served to investigate the activation of AKT and STAT5 induced by RIPC following I/R injury. As previously described above, the surgeries were performed during our previous study and only the heart tissue from the respective animals was obtained for the present study (16). To facilitate the understanding of the experimental protocol, the procedure is briefly outlined below. The rats received sham I or RIPC treatment followed by I/R or a time matched sham II treatment without any intervention (TP 2:215 min). After reperfusion, in the groups which received I/R treatment, the LAD was reoccluded and $5 \mathrm{ml}$ Evans blue solution was injected intravenously. The non area of risk (non-AAR) was stained blue, while the area of risk (AAR) remained unstained. The hearts were removed, separated in non-AAR and ARR, respectively frozen in liquid nitrogen and stored at $-80^{\circ} \mathrm{C}$. For further analyses, only tissue from the AAR was used. In the groups that did not receive I/R treatment, the hearts were removed after the matched TP, frozen in liquid nitrogen and stored at $-80^{\circ} \mathrm{C}$. The experimental groups were as follows ( $n=6 /$ group): i) Sham late TP (TP2) group, in which the rats received sham I and II treatment; ii) RIPC TP2 group, in which rats received RIPC treatment with 4 cycles of 5 min bilateral hind limb ischemia interspersed with 5 min of reperfusion, followed by sham II treatment; iii) I/R group, in which rats received sham I treatment and I/R treatment with 35 min of LAD occlusion and $120 \mathrm{~min}$ of reperfusion; and iv) RIPC $+\mathrm{I} / \mathrm{R}$ group, in which the rats received RIPC treatment with 4 cycles of 5 min bilateral hind limb ischemia interspersed with $5 \mathrm{~min}$ of reperfusion and 


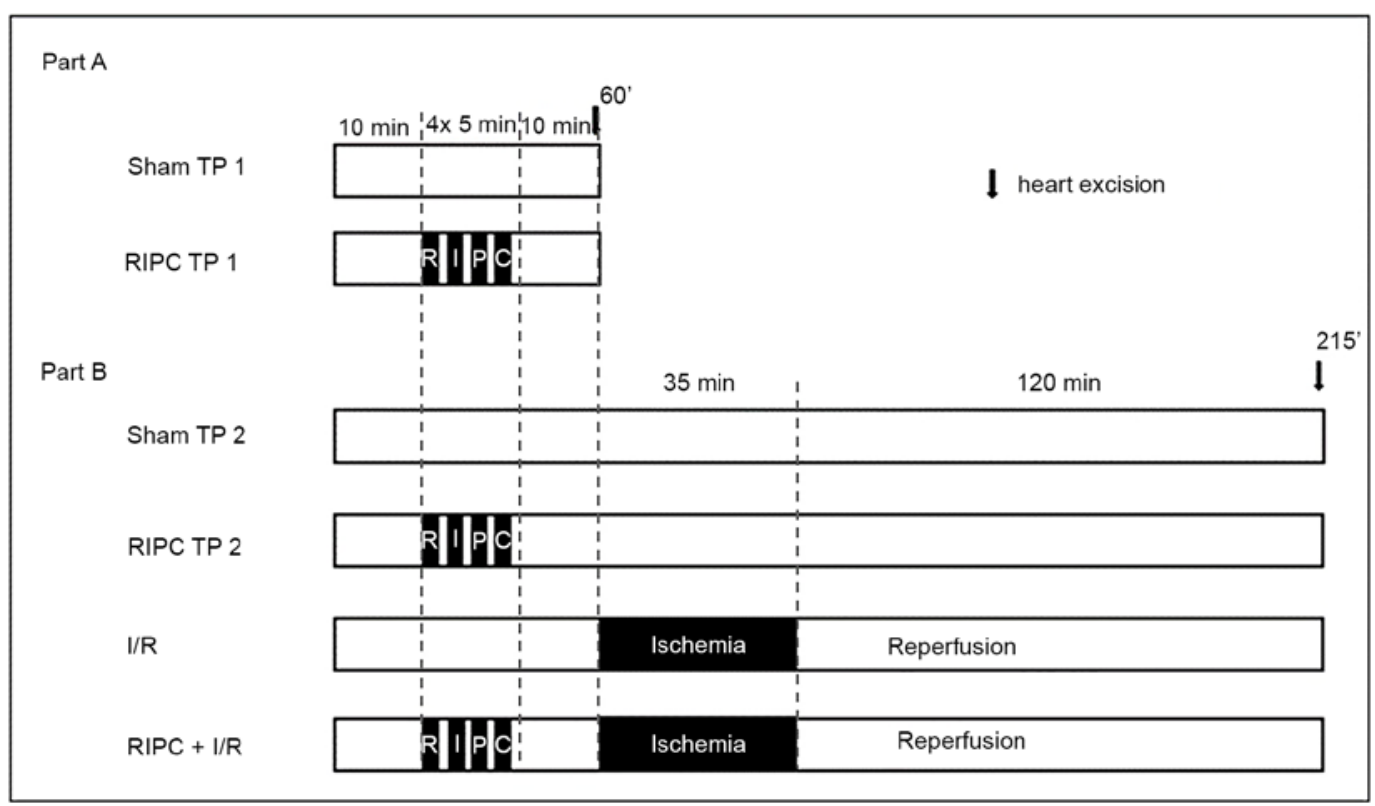

Figure 1. Experimental protocol for in vivo experiments of Part A and Part B. TP, time-point; RIPC, remote ischemic preconditioning; I/R, ischemia/reperfusion.

I/R treatment with 35 min of ischemia (LAD occlusion) and $120 \mathrm{~min}$ of reperfusion.

Protein extraction and subcellular fractioning. Tissue homogenization and protein extraction were performed as previously described (16). Briefly, pulverized, frozen heart tissue was dissolved in lysis buffer containing $5 \mathrm{mM}$ Tris base, $2 \mathrm{mM}$ EGTA, phosphatase inhibitors $(50 \mathrm{mM} \mathrm{NaF}$ and $2 \mathrm{mM}$ $\mathrm{Na}_{3} \mathrm{VO}_{4}$ ), complete protease inhibitor mix (Roche Diagnostics) and $5 \mathrm{mM}$ DTT. The lysates were homogenized and centrifuged at $600 \mathrm{x} \mathrm{g}$ at $4^{\circ} \mathrm{C}$ for $10 \mathrm{~min}$ to discard the cell debris.

Western blotting. Western blotting was performed as described previously (16). Briefly, the protein concentration was determined using the Lowry method and proteins were dissolved in loading buffer containing mercaptoethanol. Following the seperation of the proteins via $10 \%$ SDS-PAGE, the proteins were subsquently transferred onto PVDF membranes and blocked with $5 \%$ milk powder solution. The membranes were then incubated with the following primary antibodies in $1 \%$ milk powder solution overnight: Anti-GAPDH (1:5,000; cat. no. ab8245; Abcam), anti-AKT (1:1,000; cat. no. 9272; Cell Signaling Technology, Inc.), anti-phosphorylated (phospho)-AKT-serine (Ser) ${ }^{473}$ (1:1,000; cat. no. 9271; Cell Signaling Technology, Inc.), anti-phospho-STAT5-tyrosine ${ }^{694}$ (1:1,000; cat. no. 4322; Cell Signaling Technology, Inc.) and anti-STAT5 (1:1,000; cat. no. 9363; Cell Signaling Technology, Inc.). Following the primary antibody incubation, the membranes were incuabted with HRP-conjugated secondary antibodies (Jackson ImmunoResearch Laboratories, Inc.) for $2 \mathrm{~h}$. Protein bands were visualized using an enhanced chemiluminescence system (Santa Cruz Biotechnology, Inc.), and captured using a CoolSNAP HQ2 camera (Teledyne Photometrics) and a Gel-Pro analyzer 6.0.0.349 (Media Cybernetics, Inc.). Densitometric analysis was performed using Image Studio Lite version 5.2.5 software (LI-COR Biosciences). All signal intensities were normalized to the respective signaling intensity of the loading control, GAPDH. Signal intensities of the phosphorylated proteins were normalized to the respective total protein signal intensity.

Statistical analysis. The sample size used (n=6/group) was determined in order to detect the mean difference of $25 \%$ with a standard deviation of $15 \%$ in the phosphorylation levels of target proteins with $\alpha<0.05$ and a power of 0.8 (17). To detect the statistical differences in the phosphorylation levels between the two groups, a Student's t-test was performed using GraphPad Prism 6 software (GraphPad Software, Inc.). Data are expressed as the mean $\pm \mathrm{SD}$. $\mathrm{P}<0.05$ was considered to indicate a statistically significant difference.

\section{Results}

Analysis of AKT expression levels. A previous study revealed that RIPC treatment, as used in the present study, significantly reduced the infarct size (16). Therefore, to analyze the role of AKT in the present study, the levels of AKT phosphorylation at $\mathrm{Ser}^{473}$ were immediately determined following RIPC treatment (TP1) or after a subsequent I/R phase (TP2). As shown in Fig. 2, RIPC treatment did not significantly alter the phosphorylation levels of AKT compared with the sham-operated control animals at TP1 $(0.93 \pm 0.73$ vs. $0.71 \pm 0.57 ; \mathrm{P}=0.57$; Fig. $2 \mathrm{~A})$ or TP2 $(0.15 \pm 0.12$ vs. $0.13 \pm 0.05 ; \mathrm{P}=0.77$; Fig. $2 \mathrm{~B})$. Furthermore, no significant differences were identified in the AKT phosphorylation levels between the I/R group (1.36 \pm 1.07$)$ and RIPC + I/R group (2.12 \pm 1.30$)(\mathrm{P}=0.300$; Fig. 2C).

Analysis of STAT5 expression levels. To determine the involvement of STAT5 in RIPC-induced cardioprotection, the expression levels of STAT5 were determined to verify a possible translocation from the cytosol into the nuclei or mitochondria. At the TP1, STAT5 expression levels were not significantly altered following RIPC treatment compared 
A
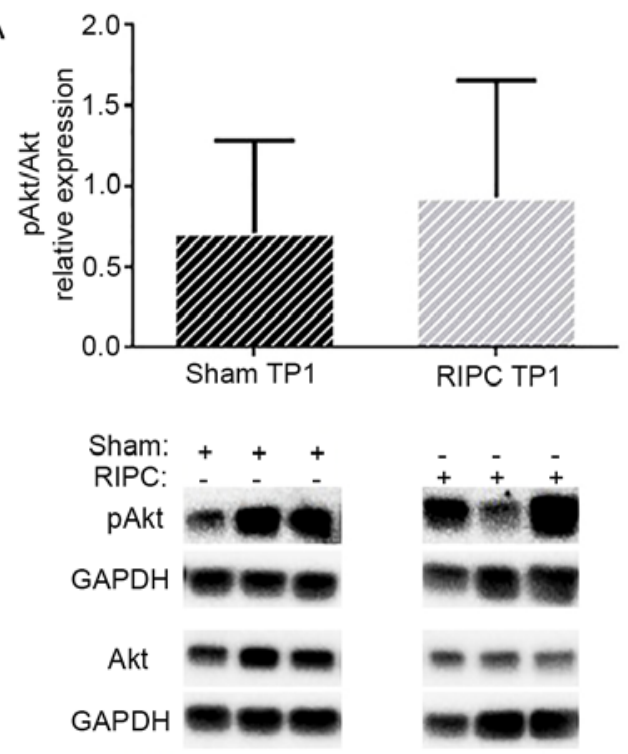

C
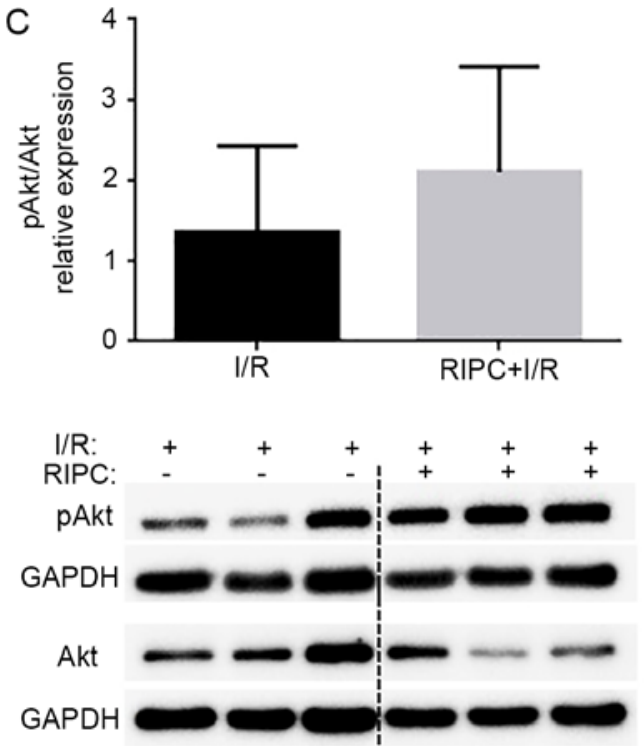

B
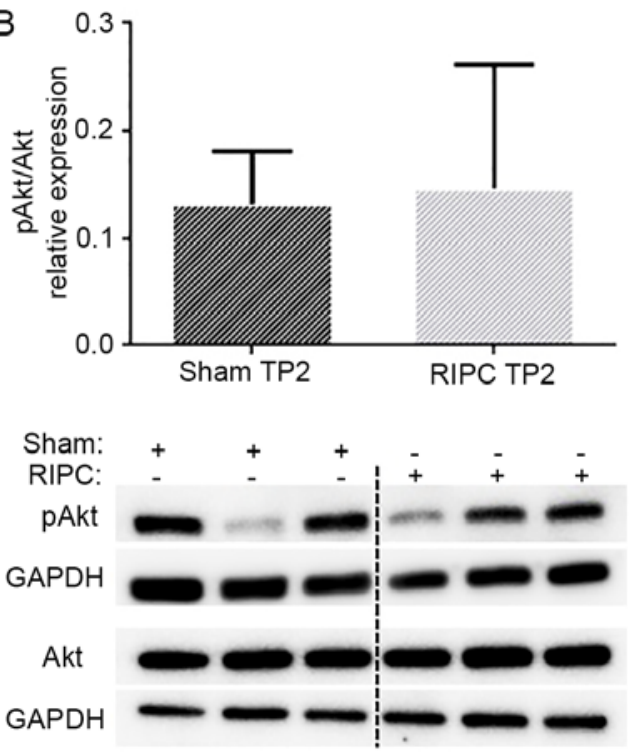

Figure 2. Analyis of phosphorylated Akt at $\operatorname{Ser}^{473}$ (pAKT). (A) Quantification of phosphorylated Akt levels at the early time-point (TP1) for Sham and RIPC group. (B) Quantification of phosphorylated Akt levels at the late time-point (TP2) for Sham and RIPC group. (C) Quantification of phosphorylated Akt levels at the late time-point (TP2) for I/R and RIPC + I/R group. Exemplary western blot analysis are depicted under each quantification showing signals from 3 representative animals per respective group. Data are presented as the means \pm standard deviation, $\mathrm{n}=6$. $\mathrm{RIPC}$, remote ischemic preconditioning; $\mathrm{I} / \mathrm{R}$, ischemia/reperfusion; TP, time-point.

with the sham-treated group $(0.79 \pm 0.33$ vs. $1.29 \pm 0.54$; $\mathrm{P}=0.09$; Fig. 3A1). At the TP2, RIPC also did not influence STAT5 expression levels compared with the sham group $(0.51 \pm 0.12$ vs. $0.60 \pm 0.20 ; \mathrm{P}=0.38$; Fig. $3 \mathrm{~B} 1)$. Furthermore, RIPC followed by I/R did not alter the STAT5 expression levels compared with the I/R group $(0.53 \pm 0.40$ vs. $0.73 \pm 0.21$; $\mathrm{P}=0.31$; Fig. $3 \mathrm{C} 1$ ).

To investigate the role of STAT5 activation by RIPC, the phosphorylation levels of STAT5 were determined. At the TP1, no significant differences were reported in the phosphorylation levels of STAT5 between the sham and RIPC groups $(0.27 \pm 0.08$ vs. $0.25 \pm 0.07 ; \mathrm{P}=0.70$; Fig. $3 \mathrm{~A} 2)$. At the TP2, neither RIPC treatment alone $(0.03 \pm 0.01 ; \mathrm{P}=0.17$; Fig. 3B2) nor RIPC followed by I/R (I/R, $0.26 \pm 0.12 \mathrm{vs}$. RIPC + I/R, $0.34 \pm 0.10 ; \mathrm{P}=0.22$; Fig. $3 \mathrm{C} 2$ ) influenced the phosphorylation levels of STAT5.

\section{Discussion}

The present study aimed to elucidate whether the activation of AKT and/or STAT5 served a role in RIPC at the investigated TPs. The results of the current study indicated that neither the activation of AKT nor STAT5 were modulated by RIPC with or without subsequent I/R, suggesting that the activation of the respective targets at the measured TPs may not be involved in RIPC-induced cardioprotection.

Focusing on the activation of AKT, the present findings revealed that the cardioprotective signaling induced by RIPC was not mediated through the phosphorylation of AKT, at least at the chosen TPs of measurement in the present study. At the TP1, in contrast to the findings of the present study, previous studies have described the activation of AKT by RIPC. For example, in an in vivo mouse model, increased levels of 
A1

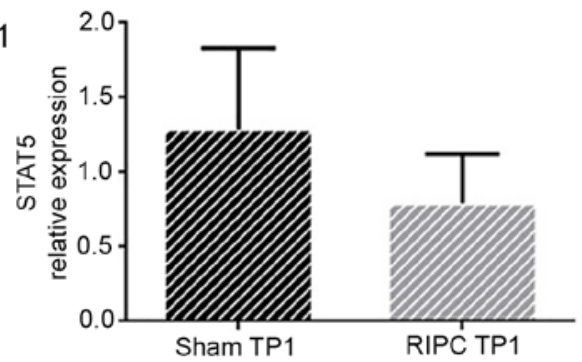

A2

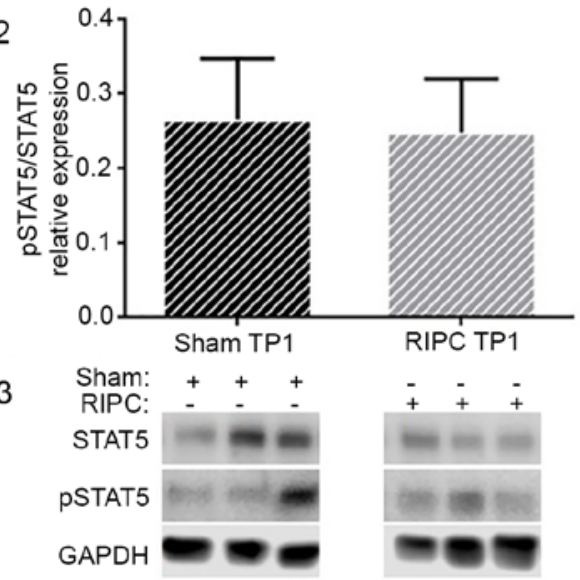

C1

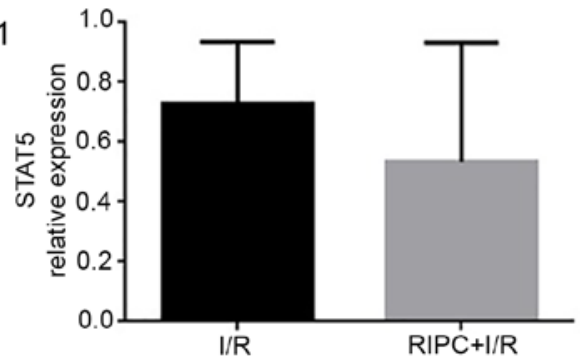

$\mathrm{C} 2$
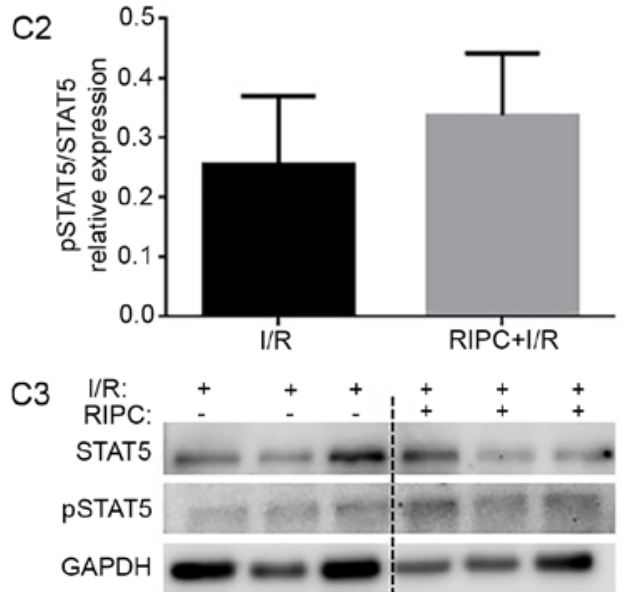

B1

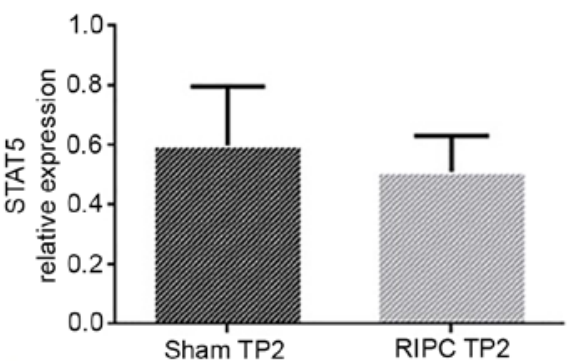

B2

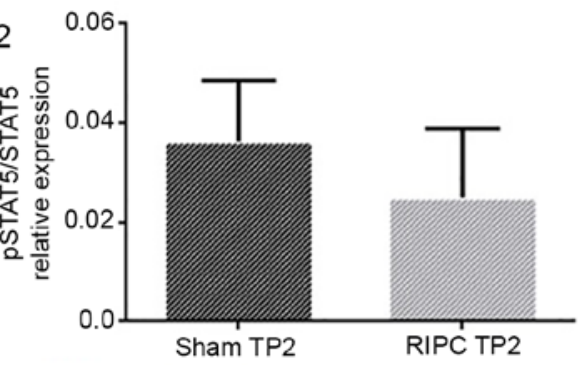

B3 $\underset{\text { SIPC }}{\text { Sham }}$

STAT5

pSTAT5

GAPDH

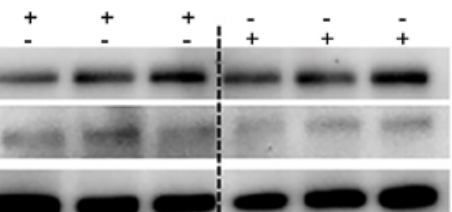

Figure 3. Expression analysis of STAT5 and phosphorylated STAT5 at Tyr ${ }^{694}$ (pSTAT). (A) Sham and RIPC group at early time-point (TP1): Quantification of STAT5 (A1) and pSTAT5 (A2) protein levels and exemplary western blot analysis (A3) showing signals from 3 representative animals per respective group. (B) Sham and RIPC group at late time-point (TP2): Quantification of STAT5 (B1) and pSTAT5 (B2) protein levels and exemplary western blot analysis (B3) showing signals from 3 representative animals per respective group. (C) I/R and RIPC + I/R group at late time-point (TP2): Quantification of STAT5 (C1) and pSTAT5 (C2) protein levels and western blot analysis (C3) demonstrating signals from 3 representative animals per respective group. Data are mean \pm standard deviation, $\mathrm{n}=6$. RIPC, remote ischemic preconditioning; $\mathrm{I} / \mathrm{R}$, ischemia/reperfusion; $\mathrm{TP}$, time-point.

phosphorylated AKT were detected 15 min after non-invasive RIPC treatment (8), while invasive RIPC via femoral artery occlusion induced the phosphorylation of AKT immediately following intervention in a rat model (18). These controversial findings suggested that the phosphorylation of AKT requires $>10$ min or a more pronounced stimulus, such as invasive RIPC, to possibly reduce this time span following intervention. In addition, species-specific differences may also be responsible 
for the different results. In pigs, Skyschally et al (19) reported no differences in the phosphorylation levels of AKT between the control and RIPC group following $1 \mathrm{~h}$, indicating that the activation of AKT may only be transient.

With regards to the TP2, similar to the results of the present study, Hildebrandt et al (14) detected no changes in the phosphorylation levels of AKT $2 \mathrm{~h}$ after reperfusion in a translational protocol transferring human RIPC plasma, collected from $5 \mathrm{~min}$ to $6 \mathrm{~h}$ after treatment, onto in vitro mouse hearts prior to I/R. These results supported the findings from the current study regarding the TP2 (following $2 \mathrm{~h}$ of reperfusion), indicating that the activation of AKT does not occur at this TP. Nonetheless, the selected TP2 during reperfusion in the current experimental protocol may have been too late for detecting AKT phosphorylation levels. In accordance with this hypothesis, Ma et al (20) observed an increase in the phosphorylated levels of AKT by RIPC 5 min after the onset of reperfusion. Taking into account the fact that increased levels of AKT phosphorylation are detectable immediately at the beginning of reperfusion may suggest that the selected TP $(2 \mathrm{~h}$ after the begining of reperfusion) may be too late to succesfully investigate AKT phosphorylation levels. Interestingly, postconditionig with RIPC or transfering RIPC plasma between species (for example, pig plasma into rat hearts) enabled the detection of AKT during the late periods of reperfusion. In addition, inducing a postconditioning stimulus with RIPC led to the activation of AKT, with even higher levels of phosphorylated AKT observed $3 \mathrm{~h}$ after the onset compared with at the beginning of reperfusion (21). Elevated Akt phosphorylation levels were also detected following $2 \mathrm{~h}$ of reperfusion through using a translational cardioprotective strategy of RIPC plasma transfer (19). In summary, the activation of AKT by RIPC seems to be dependent on the TPs chosen for measurements, the species and the stimulus.

The present study also detected no significant differences in the phosphorylation levels of STAT5 at the measured TPs. Previous studies have demonstrated comparable results; for example, the transfer of human RIPC plasma to mouse hearts or from pigs to rat hearts in the respective models of I/R injury identified no significant differences in the phosphorylation levels of STAT5 compared with the control plasma $(14,19)$. In contrast, RIPC-induced decreased levels of phosphorylated STAT5 were detected $10 \mathrm{~min}$ after the onset of reperfusion compared with in the pre-ischemia in pigs (19). Despite these controversial results, it is hypothesized that the activation of STAT5 may serve a role in RIPC-induced cardioprotection. The induction of RIPC $24 \mathrm{~h}$ before I/R injury was discovered to be cardioprotective and induce the phosphorylation of STAT5; however, both effects were abolished in a cardiomyocyte-specific STAT5 knockout mouse (12). Contrary to these findings, it seems that the activation of STAT5 serves a rather minor role in the experimental setting of cardioprotection in animal models, in contrast to its importance in the clinical setting for patients. For example, Heusch et al (11) suggested that in human hearts, cardioprotection was associated with STAT5, but not STAT3, whereas STAT3 activation and possibly STAT5 inhibition were associated with protection in animals (11). Recent clinical studies have also supported these findings, demonstrating that the phosphorylation of STAT5 was enhanced by RIPC shortly after reperfusion $(10,22)$.
Therefore, the inability to detect the activation of STAT5 in the current rat model may have been be due to species-dependent differences in signal transcription factors.

It is widely accepted that RIPC treatment induces a signal (e.g. humoral factors) release at the conditioned organ or tissue into the blood stream (5). After reaching the target organ (heart), these humoral factors confer cardioprotective properties through activating signaling cascades (5). Thus, a whole organism is essential to study these mechanisms induced by RIPC treatment and therefore, we selected an in vivo rat model of I/R injury for the present study.

There are several limitations to the present study. Firstly, the applied protocol for I/R, more specifically, concerning the time intervals of $I / R$, has been successfully established for analyzing the effects on infarct size and hemodynamics; however, it may not be sufficient for detecting the phosphorylation levels of AKT and STAT5. Therefore, a possible activation of the respective targets at different TPs following RIPC with or without $\mathrm{I} / \mathrm{R}$ is conceivable and cannot be clarified with the present experimental settings. Furthermore, the possible activation of AKT via its second phosphorylation site, threonine (Thr) ${ }^{308}$, was not analyzed. However, all other previous studies discussed in this manuscript also only analyzed the phosphorylation levels at $\mathrm{Ser}^{473}$. Therefore, the additional detection of AKT phosphorylation levels at $\mathrm{Thr}^{308}$ should be analyzed in future studies. In addition, due to the study design, an analysis of the time effects within groups was not possible. Finally, a possible translocation of phosphorylated STAT5 into the nucleus or mitochondria was not investigated in the present study, but it seems to be of great interest with regards to the modulation of energy metabolism during cardioprotection.

In conclusion, the findings of the present study using an in vivo rat model revealed that neither the phosphorylation of AKT nor STAT5 mediated the cardioprotective effects of RIPC at the measured TPs, that is, 10 min after RIPC treatment or $2 \mathrm{~h}$ after reperfusion. Due to the controversial findings in the previous studies, the association between the possible transient activation of phosphorylated AKT/STAT5 and the cardioprotective properties needs to be investigated comprehensively in future studies.

\section{Acknowledgements}

In partial fulfillment of the requirements for an MD thesis (Christian Reiter).

\section{Funding}

No funding was received.

\section{Availability of data and materials}

The datasets used and/or analyzed during the current study are available from the corresponding author on reasonable request.

\section{Authors' contributions}

AR analyzed and interpreted the data and wrote the manuscript. KF helped to analyze the data and wrote the manuscript. $\mathrm{NH}$ performed the in vivo experiments. CR performed the 
western blot analysis. TB helped to analyze and interpret the data. AH helped to design the study. MWH helped to analyze and interpret the data. RH helped to design the study, analyzed and interpreted the data and wrote the manuscript. CT analyzed and interpreted the data and wrote the manuscript. All authors read and approved the final manuscript.

\section{Ethics approval and consent to participate}

The current study was performed after obtaining approval from the State Agency for Nature, Environment and Consumer Protection (LANUV), North-Rhine Westphalia, Germany (8.87-50.10.37.09.148). The current study complies with the ARRIVE guidelines.

\section{Patient consent for publication}

Not applicable.

\section{Competing interests}

The authors declare that they have no competing interests.

\section{References}

1. Global Health Estimates 2016: Deaths by Cause, Age, Sex, by Country and by Region, 2000-2016. Geneva, World Health Organization, 2018.

2. Yellon DM and Hausenloy DJ: Myocardial reperfusion injury. N Engl J Med 357: 1121-1135, 2007.

3. Ferdinandy P, Hausenloy DJ, Heusch G, Baxter GF and Schulz R: Interaction of risk factors, comorbidities, and comedications with ischemia/reperfusion injury and cardioprotection by preconditioning, postconditioning, and remote conditioning. Pharmacol Rev 66: 1142-1174, 2014.

4. Murry CE, Jennings RB and Reimer KA: Preconditioning with ischemia: A delay of lethal cell injury in ischemic myocardium. Circulation 74: 1124-1136, 1986.

5. Heusch G: Molecular basis of cardioprotection: signal transduction in ischemic pre-, post-, and remote conditioning. Circ Res 116: 674-699, 2015.

6. Cho YJ and Kim WH: Perioperative cardioprotection by remote ischemic conditioning. Int J Mol Sci 20: 4839, 2019.

7. Rossello $\mathrm{X}$ and Yellon DM: The RISK pathway and beyond. Basic Res Cardiol 113: 2, 2017.

8. Li J, Xuan W, Yan R, Tropak MB, Jean-St-Michel E, Liang W, Gladstone R, Backx PH, Kharbanda RK and Redington AN: Remote preconditioning provides potent cardioprotection via $\mathrm{PI} 3 \mathrm{~K} / \mathrm{Akt}$ activation and is associated with nuclear accumulation of $\beta$-catenin. Clin Sci (Lond) 120: 451-462, 2011.
9. Hadebe N, Cour M and Lecour S: The SAFE pathway for cardioprotection: Is this a promising target? Basic Res Cardiol 113: 9, 2018.

10. Wu Q, Wang T, Chen S, Zhou Q, Li H, Hu N, Feng Y, Dong N, Yao S and Xia Z: Cardiac protective effects of remote ischaemic preconditioning in children undergoing tetralogy of fallot repair surgery: A randomized controlled trial. Eur Heart J 39: 1028-1037, 2018

11. Heusch G, Musiolik J, Kottenberg E, Peters J, Jakob H and Thielmann M: STAT5 activation and cardioprotection by remote ischemic preconditioning in humans: Short communication. Circ Res 110: 111-115, 2012.

12. Chen H, Jing XY, Shen YJ, Wang TL, Ou C, Lu SF, Cai Y, Li Q, Chen X, Ding YJ, et al: Stat5-dependent cardioprotection in late remote ischaemia preconditioning. Cardiovasc Res 114: 679-689, 2018.

13. Weber NC, Riedemann I, Smit KF, Zitta K, van de Vondervoort D, Zuurbier CJ, Hollmann MW, Preckel B and Albrecht M: Plasma from human volunteers subjected to remote ischemic preconditioning protects human endothelial cells from hypoxia-induced cell damage. Basic Res Cardiol 110: 17, 2015.

14. Hildebrandt HA, Kreienkamp V, Gent S, Kahlert P, Heusch G and Kleinbongard P: Kinetics and signal activation properties of circulating factor(s) from healthy volunteers undergoing remote ischemic pre-conditioning. JACC Basic Transl Sci 1: 3-13, 2016.

15. Kilkenny C, Browne WJ, Cuthill IC, Emerson M and Altman DG: Improving bioscience research reporting: The ARRIVE guidelines for reporting animal research. PLoS Biol 8: e1000412,2010.

16. Brandenburger T, Huhn R, Galas A, Pannen BH, Keitel V, Barthel F, Bauer I and Heinen A: Remote ischemic preconditioning preserves connexin 43 phosphorylation in the rat heart in vivo. J Transl Med 12: 228, 2014.

17. Chow SC, Shao J and Wang H: Sample Size Calculations in Clinical Research. 2nd edition. Chapman \& Hall/CRC Biostatistics Series, pp58. 2008.

18. Donato M, Goyeneche MA, Garces M, Marchini T, Pérez V, Del Mauro J, Höcht C, Rodriguez M, Evelson P and Gelpi RJ: Myocardial triggers involved in activation of remote ischaemic preconditioning. Exp Physiol 101: 708-716, 2016.

19. Skyschally A, Gent S, Amanakis G, Schulte C, Kleinbongard P and Heusch G: Across-species transfer of protection by remote ischemic preconditioning with species-specific myocardial signal transduction by reperfusion injury salvage kinase and survival activating factor enhancement pathways. Circ Res 117: 279-288, 2015.

20. Ma LL, Kong FJ, Guo JJ, Zhu JB, Shi HT, Li Y, Sun RH and Ge JB: Hypercholesterolemia abrogates remote ischemic preconditioning-induced cardioprotection: Role of reperfusion injury salvage kinase signals. Shock 47: 363-369, 2017.

21. Hong J, Ge HW, Liu JQ, Sun RH and Kong FJ: Pharmacological inhibition of PTEN restores remote ischemic postconditioning cardioprotection in hypercholesterolemic mice: Potential role of PTEN/AKT/GSK3 3 SIGNALS. Shock 52: 522-531, 2019.

22. Gedik N, Thielmann M, Kottenberg E, Peters J, Jakob H, Heusch G and Kleinbongard P: No evidence for activated autophagy in left ventricular myocardium at early reperfusion with protection by remote ischemic preconditioning in patients undergoing coronary artery bypass grafting. PLoS One 9: e96567, 2014. 